\title{
Neuroprotective effects of ceftriaxone: insights from in vitro and in vivo models
}

\author{
M.A. Tikhonova ${ }^{1,2 *}$, T.G. Amstislavskaya ${ }^{1,2}$, H. Kunugi ${ }^{3}$, Y.J. Ho $^{4}$ \\ ${ }^{1}$ Scientific Research Institute of Physiology and Basic Medicine, Novosibirsk, Russia \\ ${ }^{2}$ Novosibirsk State University, Novosibirsk, Russia \\ ${ }^{3}$ National Center of Neurology and Psychiatry, Tokyo, Japan \\ ${ }^{4}$ Chung Shan Medical University, Taichung City, Taiwan, R.O.C. \\ *e-mail: tikhonovama@physiol.ru
}

Key words: cognitive deficits, neuroinflammation, mice, rats, Parkinson's disease, Alzheimer's disease, cortical neurons

Motivation and Aim: Drug repurposing appeared to be quite effective strategy in psychopharmacology. Ceftriaxone (CEF) is a safe and multipotent agent that has been used for decades as an antimicrobial drug. Recently the data were obtained on its neuroprotective properties. We obtained promising results on its neuroprotective properties including the restoration of cognitive deficits in MPTP-induced rat model of Parkinson's disease. The aim of this study was to determine the potential effects of CEF to restore cognitive deficits in animal models of Alzheimer's disease (AD) as well as to reveal neuronal mechanisms in the effects.

Methods and Algorithms: Primary culture of rat cortical neurons was used to evaluate cell survival against $\mathrm{H} 2 \mathrm{O} 2(50 \mu \mathrm{M})$ in MTT test after CEF treatment (100 $\mu \mathrm{M}, 1 \mathrm{x}, 5$ days prior MTT test). Western-blotting was applied to estimate molecular changes. Mice of C57Bl/6J strain injected bilaterally i.c.v. with amyloid-beta fragment 25-35 were used as pharmacological AD model while rats of OXYS strain were used as a genetic model of sporadic AD. To evaluate the effects of CEF, the animals of experimental groups were treated with the drug $(100 \mathrm{mg} / \mathrm{kg} /$ day, i.p., 36 days $)$ and then underwent behavioral testing for cognitive function and their brains were assessed with Nissl staining and immunohistochemical analysis.

Results: CEF treatment exhibited beneficial effects on some of impaired cognitive features in OXYS rats and mice with deficits induced by amyloid-beta. Neuromorphologically, CEF increased the density of pyramidal neurons in the hippocampal CA1 area, decreased the number of degenerating neurons and edema of brain tissue in OXYS rats. It also decreased amyloid accumulation in mice. CEF significantly increased cell survival of cortical neurons in vitro. This effect was associated with a decrease in pAkt and pERK levels.

Conclusion: The results suggest CEF as a promising pharmacological tool for the prevention of cognitive decline at neurodegenerative disorders and gave new insights into mechanisms of its neuroprotective effects.

Acknowledgements: Supported partially by grant No. 15-04-05593-a from the Russian Foundation for Basic Research (Russia), by NSU: Academic Strategic Unit "Neuroscience in Translational Medicine”, and by grant No. L-15569 from JSPS (Japan). The studies were partially implemented using the Unique scientific installation "Biological collection - Genetic biomodels of neuro-psychiatric disorders" (No. 493387) at Scientific Research Institute of Physiology and Basic Medicine. 